\title{
Catalytic pyrolysis of the Empty Fruit Bunches of the Oil Palm and the Sugarcane Bagasse: Non-Isothermal Thermogravimetric Kinetic Analysis
}

\author{
Victor Pretell, $\mathrm{MSc}^{1}$, Raymundo Erazo, $\mathrm{Dr}^{2}$ \\ ${ }^{1}$ Universidad Nacional de Ingeniería, Perú, vpretell@fip.uni.edu.pe \\ ${ }^{2}$ Universidad Nacional Mayor de San Marcos, Perú, ray2erazo@yahoo.es
}

\begin{abstract}
The kinetics of the catalytic pyrolysis of the empty fruit bunches of the oil palm (EFB) and the sugarcane bagasse (SCB) were investigated, using the non-isothermal thermogravimetric analysis. Two isoconversional methods were applied, the Friedman differential method and the Ozawa-Flynn-Wall integral method. Five catalysts were evaluated: Equilibrium catalyst of FCC (Z2), ZSM-5 in pellet (Z3), zeolite ZSM-5 in spherical pellet (Z4), Zeolite Y powder (Z5) and zeolite ZSM-5 powder (Z6). The activation energy in the pyrolysis of the biomass with and without catalyst was calculated. Biomass with a particle size between 177 to $250 \mu \mathrm{m}$ was mixed with an equal amount of catalyst (particle size less than 250 $\mu \mathrm{m}$ ). In the thermogravimetric analyzer, it was heated from $30^{\circ} \mathrm{C}$ to $900{ }^{\circ} \mathrm{C}$ under non-isothermal conditions at four heating rates 5,10 , 15 and $20{ }^{\circ} \mathrm{C} / \mathrm{min}$ respectively, using nitrogen at $60 \mathrm{~cm} 3 / \mathrm{min}$ as inertizing gas. Regarding the SCB, it was obtained that the catalyst $\mathrm{Z2} \mathrm{had} \mathrm{the} \mathrm{lowest} \mathrm{activation} \mathrm{energy} \mathrm{of} 123.26 \mathrm{~kJ} / \mathrm{mol}$ (OFW) and $123.91 \mathrm{~kJ} / \mathrm{mol}$ (Friedman) and the BCA without catalyst $139.57 \mathrm{~kJ} /$ mol (OFW) and $147.88 \mathrm{~kJ} / \mathrm{mol}$ (Friedman) which indicates that it is the most useful for catalytic pyrolysis of SCB. In the pyrolysis of the $E F B$, activation energy values of $158.19 \mathrm{~kJ} / \mathrm{mol}(\mathrm{OFW})$ and 146.56 $\mathrm{kJ} / \mathrm{mol}$ (Friedman) were obtained without catalyst and that catalyst $\mathrm{Z} 2 \mathrm{had}$ the lowest values of activation energy, $114.48 \mathrm{~kJ} / \mathrm{mol}(\mathrm{OFW})$ and $102.55 \mathrm{~kJ} / \mathrm{mol}$ (Friedman), which indicates that it is suitable for catalytic pyrolysis of EFB. The catalyst with the highest activation energy was Z5 for both biomass and both methods (OFW and Friedman). For the $S C B$, values of $168.47 \mathrm{~kJ} / \mathrm{mol}(\mathrm{OFW})$ and 168.42 $\mathrm{kJ} / \mathrm{mol}$ (Friedman) were obtained and for EFB $163.77 \mathrm{~kJ} / \mathrm{mol}$ $(\mathrm{OFW})$ and $189.27 \mathrm{~kJ} / \mathrm{mol}$ (Friedman), this indicates that it is not suitable for catalytic pyrolysis of SCB and EFB.
\end{abstract}

Keywords- Pyrolysis, Isoconversion, Thermogravimetric analysis, Kinetic, Catalyst.

Digital Object Identifier (DOI):

http://dx.doi.org/10.18687/LACCEI2020.1.1.69

ISBN: 978-958-52071-4-1 ISSN: 2414-6390

$1^{\text {th }}$ LACCEI International Multi-Conference for Engineering, Education, and Technology: "Engineering, Integration, and Alliances for a Sustainable Development" "Hemispheric Cooperation for Competitiveness and Prosperity on a Knowledge-Based Economy", 27-31 July 2020, Virtual Edition. 


\title{
Pirólisis Catalítica de los Racimos de Frutos Vacíos de la Palma Aceitera y el Bagazo de Caña de Azúcar: Análisis Cinético Termogravimétrico No Isotérmico
}

\author{
Victor Pretell, $\mathrm{MSc}^{1}$, Raymundo Erazo, $\mathrm{Dr}^{2}$ \\ ${ }^{1}$ Universidad Nacional de Ingeniería, Perú, vpretell@fip.uni.edu.pe \\ ${ }^{2}$ Universidad Nacional Mayor de San Marcos, Perú, ray2erazo@yahoo.es
}

\begin{abstract}
Resumen-Se investigó la cinética de la pirólisis catalítica de los racimos de frutos vacios de la palma aceitera (RFV) y del bagazo de caña de azúcar (BCA), usando el análisis termogravimétrico no isotérmico. Se aplicaron dos métodos isoconversionales el método diferencial de Friedman y el método integral de Ozawa-Flynn-Wall. Se evaluaron cinco catalizadores: Catalizador de equilibrio de FCC (Z2), ZSM-5 en pellet (Z3), zeolita ZSM-5 en pellet esférico (Z4), Zeolita Y en polvo (Z5) y zeolita ZSM-5 en polvo (Z6). Se calculo la energía de activación en la pirólisis de la biomasa con y sin catalizador. La biomasa con un tamaño de partícula entre 177 a $250 \mu m$ se mezcló con una cantidad igual de catalizador (tamaño de partícula menor a 250 $\mu \mathrm{m})$. En el analizador termogravimétrico se calentó de $30^{\circ} \mathrm{C}$ a 900 ${ }^{\circ} \mathrm{C}$ en condiciones no isotérmicas a cuatro regímenes de calentamiento $5,10,15$ y $20{ }^{\circ} \mathrm{C} / \mathrm{min}$ respectivamente, empleando nitrógeno a $60 \mathrm{~cm}^{3} / \min$ como gas inertizante. Respecto al BCA se obtuvo que el catalizador Z2 tenía la energía de activación más baja y de $123.26 \mathrm{~kJ} / \mathrm{mol}(\mathrm{OFW}$ ) y $123.91 \mathrm{~kJ} / \mathrm{mol}$ (Friedman) y el BCA sin catalizador $139.57 \mathrm{~kJ} / \mathrm{mol}(O F W)$ y $147.88 \mathrm{~kJ} / \mathrm{mol}$ (Friedman) lo que indica que es el más útil para la pirólisis catalítica del BCA. En la pirólisis de los RFV se obtuvo valores de la energía de activación de $158.19 \mathrm{~kJ} / \mathrm{mol}(\mathrm{OFW})$ y $146.56 \mathrm{~kJ} / \mathrm{mol}$ (Friedman) sin catalizador y que el catalizador Z2 tuvo los menores valores de la energía de activación, $114.48 \mathrm{~kJ} / \mathrm{mol}(\mathrm{OFW})$ y $102.55 \mathrm{~kJ} / \mathrm{mol}$ (Friedman) respectivamente, lo que indica que es el adecuado para la pirólisis catalítica de los RFV. El catalizador con mayor energía de activación fue el Z5 para ambas biomasas y ambos métodos (OFW y Friedman). Para el BCA se obtuvo valores de 168.47 $\mathrm{kJ} / \mathrm{mol}(\mathrm{OFW}$ ) y $168.42 \mathrm{~kJ} / \mathrm{mol}$ (Friedman) y para los RFV 163.77 $\mathrm{kJ} / \mathrm{mol}(\mathrm{OFW}$ ) y $189.27 \mathrm{~kJ} / \mathrm{mol}$ (Friedman).
\end{abstract}

Palabras clave- Pirólisis, Isoconversión, Análisis termogravimétrico, Cinética, Catalizador.

Abstract- The kinetics of the catalytic pyrolysis of the empty fruit bunches of the oil palm (EFB) and the sugarcane bagasse (SCB) were investigated, using the non-isothermal thermogravimetric analysis. Two isoconversional methods were applied, the Friedman differential method and the Ozawa-FlynnWall integral method. Five catalysts were evaluated: Equilibrium

Digital Object Identifier (DOI):

http://dx.doi.org/10.18687/LACCEI2020.1.1.69

ISBN: 978-958-52071-4-1 ISSN: 2414-6390 catalyst of FCC (Z2), ZSM-5 in pellet (Z3), zeolite ZSM-5 in spherical pellet (Z4), Zeolite $Y$ powder (Z5) and zeolite ZSM-5 powder (Z6). The activation energy in the pyrolysis of the biomass with and without catalyst was calculated. Biomass with a particle size between 177 to $250 \mu \mathrm{m}$ was mixed with an equal amount of catalyst (particle size less than $250 \mu \mathrm{m}$ ). In the thermogravimetric analyzer, it was heated from $30^{\circ} \mathrm{C}$ to $900{ }^{\circ} \mathrm{C}$ under non-isothermal conditions at four heating rates $5,10,15$ and $20^{\circ} \mathrm{C} / \mathrm{min}$ respectively, using nitrogen at $60 \mathrm{~cm}^{3} /$ min as inertizing gas. Regarding the $S C B$, it was obtained that the catalyst $Z 2$ had the lowest activation energy of $123.26 \mathrm{~kJ} / \mathrm{mol}(\mathrm{OFW})$ and $123.91 \mathrm{~kJ} / \mathrm{mol}$ (Friedman) and the BCA without catalyst $139.57 \mathrm{~kJ} / \mathrm{mol}(\mathrm{OFW})$ and $147.88 \mathrm{~kJ} / \mathrm{mol}$ (Friedman) which indicates that it is the most useful for catalytic pyrolysis of SCB. In the pyrolysis of the EFB, activation energy values of $158.19 \mathrm{~kJ} / \mathrm{mol}(\mathrm{OFW})$ and $146.56 \mathrm{~kJ} / \mathrm{mol}$ (Friedman) were obtained without catalyst and that catalyst Z2 had the lowest values of activation energy, $114.48 \mathrm{~kJ} / \mathrm{mol}(\mathrm{OFW})$ and 102.55 $\mathrm{kJ} / \mathrm{mol}$ (Friedman), which indicates that it is suitable for catalytic pyrolysis of EFB. The catalyst with the highest activation energy was Z5 for both biomass and both methods (OFW and Friedman). For the SCB, values of $168.47 \mathrm{~kJ} / \mathrm{mol}(\mathrm{OFW})$ and $168.42 \mathrm{~kJ} / \mathrm{mol}$ (Friedman) were obtained and for EFB $163.77 \mathrm{~kJ} / \mathrm{mol}(\mathrm{OFW})$ and $189.27 \mathrm{~kJ} / \mathrm{mol}$ (Friedman), this indicates that it is not suitable for catalytic pyrolysis of SCB and EFB.

Keywords- Pyrolysis, Isoconversion, Thermogravimetric analysis, Kinetic, Catalyst.

\section{INTRODUCCIÓN}

La demanda mundial de energía es creciente y para el 2040 se espera que el $85 \%$ se cubra con energías renovables y con gas natural, además las energías renovables serán la mayor fuente de generación de energía eléctrica [1]. La biomasa es una de las tres fuentes de energía primaria más abundante en el planeta después del carbón y los hidrocarburos fósiles (petróleo y gas natural).

El Perú es un país de ingresos medios que en los últimos veinte años ha tenido un crecimiento sostenido, esto se ha reflejado en el campo de los cultivos agroindustriales para exportación y uso doméstico. Cultivos la palma aceitera y la 
caña de azúcar que en el año 2000 producían 181,155 toneladas y 7.13 millones de toneladas, al año 2018 produjeron 921,001 toneladas y 11.72 millones de toneladas respectivamente, esto ha significado prácticamente quintuplicar la producción de palma aceitera y casi duplicar la producción de caña de azúcar. Este crecimiento trae aparejada la producción de grandes cantidades de residuos lignocelulósicos como los racimos de frutos vacíos de la palma aceitera (RFV) y el bagazo de caña de azúcar (BCA), que pueden originar problemas de tipo ambiental y de salud como la propagación de enfermedades en los cultivos [2]. Esta biomasa residual es una carga atractiva como fuente potencial de hidrocarburos y productos químicos con casi cero emisiones de dióxido de carbono [3].

La pirólisis es un proceso termoquímico donde la biomasa es sometida a calentamiento en ausencia de oxígeno y puede ser convertida en productos gaseosos, líquidos (tar) y sólidos (char), estos productos pueden ser usados como combustibles o precursores para la obtención de productos químicos, las ventajas de la pirólisis respecto a la gasificación y combustión es que tiene menores requerimientos de temperatura, que procede en ausencia de oxígeno y da productos líquidos de alta calidad [3], [4], [5], [6], pese a ello la transformación de la biomasa por pirólisis es altamente dependiente de la cinética de las reacciones de pirólisis, por eso es necesario conocer la cinética de la pirólisis de la biomasa para poder diseñar, modelar y optimizar adecuadamente el reactor, equipo clave en los procesos a escala industrial [7].

Las zeolitas son un tipo de aluminosilicato cristalino con poros homogéneos y estructura tridimensional, formado por tetraedros de silicio y aluminio que están coordinados por puentes de oxígeno no lineales. Debido a sus propiedades acidas y a la selectividad de forma se aplican en la pirólisis catalítica de la biomasa lignocelulósica y por ello están siendo estudiadas extensamente. Las zeolitas como la ZSM-5 son efectivas para convertir los compuestos altamente oxigenados contenidos en el biopetróleo en aromáticos como el benceno, tolueno y xileno (BTX). El catalizador de equilibrio de los procesos de craqueo catalítico fluidizado que se emplean en la refinación del petróleo esta básicamente compuestos por zeolita-Y con cantidades variables de aditivos que pueden ser ZSM-5, cada cierto periodo de tiempo se retira una cantidad del catalizador para ser reemplazado por catalizador fresco porque el catalizador va perdiendo parte de su actividad catalítica ya que se depositan coque y metales sobre su superficie. Se estima que en una unidad de craqueo catalítico fluidizado (FCC) se puede generar entre 300-600 ton/año de catalizador gastado [8], [9].

La cinética de la pirólisis de la biomasa es comúnmente estudiada por análisis termogravimétrico (TGA), esta técnica permite medir la pérdida de masa versus el tiempo o la temperatura en una atmosfera sin oxígeno y a una velocidad de calentamiento constante. El análisis termogravimétrico se puede desarrollar a condiciones isotérmicas o no isotérmicas. Se prefieren las condiciones no isotérmicas porque pese a que son más sensibles al ruido experimental son menos susceptibles a los errores en la medición de la masa, y también se requieren menos datos que en el caso del análisis isotérmico [10], [11]. La Confederación Internacional para Análisis Térmicos y Calorimetría (ICTAC) a través de su comité de cinética ha establecido una serie de lineamientos para la obtención de datos cinéticos validos que se centran en el control de la temperatura, velocidad de calentamiento, tipo y flujo de la atmosfera gaseosa y presión que se establecen en el equipo y la forma y tamaño de la muestra a procesar [12]. El propósito del análisis cinético desde el punto de vista computacional es establecer relaciones matemáticas entre la velocidad de descomposición de la biomasa, la conversión y la temperatura, para ello la forma más directa es determinar el triplete cinético: Energía de activación (Ea), factor de frecuencia (A) y modelo de la reacción $\mathrm{f}(\alpha)$ [13]. Para ello hay varios métodos que se pueden aplicar, los cuales se pueden clasificar en dos tipos generales: a) ajuste de las funciones y b) cinética libre (isoconversional). En el primer caso los datos termogravimétricos se ajustan por correlación estadística a una serie de modelos matemáticos (diferenciales o integrales) que determinan los modelos cinéticos de la reacción. En el segundo caso no se requiere suponer un modelo de la reacción y se puede determinar los parámetros cinéticos precisos basados en los datos termogravimétricos obtenidos a diferentes velocidades de calentamiento [14], [15]. El método isoconversional se subdivide en diferencial e integral, para el caso diferencial la aproximación propuesta por Friedman es la más usada; en el caso integral hay varias aproximaciones como las de Ozawa-Flynn-Wall (OFW), Kissinger-AkahiraSunose (KAS) y Starink [13].

La biomasa lignocelulósica se compone de celulosa, hemicelulosa y lignina más una pequeña cantidad de extraíbles y ceniza. La celulosa es el componente más abundante de la biomasa $(40-60 \%)$, y está compuesta de cadenas largas de unidades de glucosa, pero difiere en cada biomasa en los extremos de la cadena, grado de polimerización y la cristalinidad. La pirólisis de la celulosa ocurre entre los $315^{\circ} \mathrm{C}$ a $400{ }^{\circ} \mathrm{C}$ y la energía de activación esta entre $166-250 \mathrm{~kJ} / \mathrm{mol}$. La hemicelulosa está compuesta de heteropolisacáridos y es el 15 - $30 \%$ de la biomasa. La pirólisis se da en el rango de 220 ${ }^{\circ} \mathrm{C}$ a $315{ }^{\circ} \mathrm{C}$ y la energía de activación entre $169.7-186.8$ $\mathrm{kJ} / \mathrm{mol}$. La lignina se compone de tres unidades básicas: alcohol coniferílico, alcohol sinapílico y alcohol pcoumarilo. Estas unidades se unen y forman una estructura tridimensional amorfa que representa el 10 - $25 \%$ de la biomasa. La pirólisis de las muestras de lignina ocurre en un rango de temperatura de $44{ }^{\circ} \mathrm{C}$ a $824{ }^{\circ} \mathrm{C}$ con la energía de activación en el rango de $237.1-266.6 \mathrm{~kJ} / \mathrm{mol}$ [8], [16].

En este trabajo se investiga la cinética de la pirólisis catalítica de los racimos de frutos vacíos de la palma aceitera y del bagazo de caña de azúcar, usando el análisis termogravimétrico no isotérmico con el objetivo de comparar la actividad de catalizadores zeolíticos como la zeolita-Y, ZMS-5 y catalizador de equilibrio de FCC. 


\section{MATERIALES Y MÉTODOS}

\section{A. Muestras de biomasa}

Se obtuvieron muestras de dos biomasas residuales: $50 \mathrm{~kg}$ de bagazo adquiridos en la Empresa Agroindustrial Pomalca S.A.A. localizada en el distrito de Pomalca, provincia de Chiclayo, Perú y $50 \mathrm{~kg}$ de RFV en una plantación particular ubicada en el distrito y provincia de Lamas, Perú. Todas las muestras fueron secadas en un espacio ventilado pero techado durante 15 días hasta que la humedad fue menor al 10\%, luego fueron molidas en un molino de cuchillas hasta un tamaño de partícula menor a $850 \mu \mathrm{m}$ y posteriormente fueron tamizadas a diferentes tamaños de malla según lo requerían los análisis respectivos. Todas las muestras fueron almacenadas en recipientes herméticos y en ambiente ventilado para su uso posterior. La caracterización de la biomasa fue reportada en una publicación anterior [17], parte de ella se muestra en la Tabla I.

TABLA I

ANÁLISIS ElEMENTAL, PRÓXIMO, QUíMICO Y PODER CALORÍFICO

\begin{tabular}{|c|c|c|}
\hline \multirow[t]{2}{*}{ Parámetro } & \multicolumn{2}{|c|}{ Biomasa } \\
\hline & $\begin{array}{c}\text { Bagazo de caña } \\
\text { de azúcar }\end{array}$ & $\begin{array}{c}\text { Racimos de frutos vacíos } \\
\text { de palma aceitera }\end{array}$ \\
\hline \multicolumn{3}{|c|}{ Análisis Elemental $(\%)^{\mathrm{a}}$} \\
\hline $\mathrm{C}$ & 45.13 & 45.31 \\
\hline $\mathrm{H}$ & 6.37 & 6.46 \\
\hline $\mathrm{N}$ & 0.21 & 0.47 \\
\hline $\mathrm{S}$ & 0.05 & 0.07 \\
\hline $\mathrm{O}^{\mathrm{b}}$ & 41.07 & 41.15 \\
\hline \multicolumn{3}{|c|}{ Análisis Próximo (\%) } \\
\hline Humedad & 1.80 & 3.66 \\
\hline Ceniza & 7.04 & 6.30 \\
\hline Materia Volátil & 77.78 & 72.43 \\
\hline Carbono Fijo $^{\mathrm{c}}$ & 13.38 & 17.61 \\
\hline \multicolumn{3}{|c|}{ Análisis Químico (\%) } \\
\hline Holocelulosa & 60.13 & 63.11 \\
\hline Lignina & 26.01 & 27.75 \\
\hline Celulosa & 46.63 & 45.53 \\
\hline Hemicelulosa $a^{\mathrm{s}}$ & 13.50 & 17.58 \\
\hline \multicolumn{3}{|c|}{ Poder Calorífico (kJ/kg) } \\
\hline $\mathrm{PCS}^{\mathrm{e}}$ & 18281.79 & 17313.72 \\
\hline
\end{tabular}

${ }^{\text {a }}$ Resultados en base seca

${ }^{\mathrm{b}} \mathrm{El} \%$ oxigeno $=100-\% \mathrm{C}-\% \mathrm{H}-\% \mathrm{~N}-\% \mathrm{~S}-\% \mathrm{Ceniza}_{\text {(base seca) }}$

${ }^{\mathrm{c}}$ El carbono fijo se obtuvo por diferencia

${ }^{\mathrm{d}} \mathrm{MV}=$ Materia Volátil, $\mathrm{CF}=$ Carbono Fijo

${ }^{\text {e }}$ PCS, Poder Calorífico Superior

\section{B. Catalizadores}

Los catalizadores empleados se muestran en la Tabla II, se emplearon cinco catalizadores: a) catalizador de equilibrio de craqueo catalítico de una refinería local en la ciudad de
Lima, Perú, en general el catalizador de equilibrio esta compuesto de zeolitas HY o USY, b) zeolita ZSM-5 en pellet, zeolita ZSM-5 en polvo y zeolita Y en polvo adquiridas a ACS Material LLC y c) zeolita ZSM-5 esférica adquirida a Naike Chemical Industry Equipment Packing Co., Ltd., no se realizó ningún pretratamiento a los catalizadores antes de su uso, los pellets fueron molidos a polvo en un mortero de ágata y tamizados a un tamaño menor a $250 \mu \mathrm{m}$ cuando fue necesario. Para el análisis BET se empleó un analizador Gemini VII 2390t de Micromeritics Instrument Corporation.

TABLA II Propiedades de los CATALIZAdores Zeolíticos

\begin{tabular}{|l|c|c|c|c|c|}
\hline \multirow{2}{*}{ Catalizador } & \multicolumn{4}{|c|}{ Datos del fabricante } & $\begin{array}{c}\text { Área } \\
\text { superficial } \\
\mathrm{BET}^{\mathrm{a}} \\
\left(\mathrm{m}^{2} / \mathrm{g}\right)\end{array}$ \\
\cline { 2 - 5 } & Tipo & Forma & $\begin{array}{c}\text { Razón } \\
\text { molar, } \\
\mathrm{SiO}_{2} / \mathrm{Al}_{2} \mathrm{O}_{3}\end{array}$ & $\begin{array}{c}\text { Densidad } \\
\text { aparente } \\
\left(\mathrm{cm}^{3} / \mathrm{g}\right)\end{array}$ & 126 \\
\hline Cat. de FCC & Granular & N.D. & N.D. & 0.934 & 377 \\
\hline ZSM-5 $^{\mathrm{c}}$ & Pellet & $\mathrm{Na}^{+}$ & 360 & 0.68 & 234 \\
\hline ZSM-5 $^{\text {d }}$ & Esferas & $\mathrm{NH}_{4}^{+}$ & $25-30$ & $0.60 \sim 0.70$ & 596 \\
\hline $\mathrm{Y}^{\mathrm{c}}$ & Polvo & $\mathrm{H}^{+}$ & $>5$ & N.D. & 358 \\
\hline ZSM5 $^{\mathrm{c}}$ & Polvo & $\mathrm{H}^{+}$ & 360 & N.D. & 35 \\
\hline
\end{tabular}

${ }^{a}$ Medido a muestras de catalizador con tamaño de partícula $<250 \mu \mathrm{m}$

${ }^{\mathrm{b}}$ Catalizador de equilibrio de FCC, datos obtenidos de [18]

${ }^{\mathrm{c}}$ ACS Material LLC, USA

${ }^{d}$ Naike Chemical Industry Equipment Packing Co., Ltd., China N.D. No Disponible

TABLA III CODIFICACIÓN DE LAS MUESTRAS

\begin{tabular}{|c|c|}
\hline Código & Descripción \\
\hline BCA o RFV & Biomasa sin catalizador \\
\hline Z2 & Catalizador de equilibrio de FCC + Biomasa \\
\hline Z3 & ZSM-5 en pellet (G360) + Biomasa \\
\hline Z4 & ZSM-5 en pellet esférico + Biomasa \\
\hline Z5 & Zeolita Y (Y-RE16), polvo + Biomasa \\
\hline Z6 & ZSM-5 polvo (P-360), polvo + Biomasa \\
\hline
\end{tabular}

\section{Análisis termogravimétrico no isotérmico}

Para el análisis termogravimétrico no isotérmico se empleó un analizador TGA 4000 de Perkin Elmer. Las muestras de biomasa con un tamaño de partícula entre 177 a $250 \mu \mathrm{m}$ fueron mezcladas con una cantidad igual de catalizador (tamaño de partícula menor a $250 \mu \mathrm{m}$ ), se colocaron en un desecador por 12 horas y luego se tomó 1 a 2 mg de esa mezcla y se lo coloco en un crisol de alúmina y se calentó de $30{ }^{\circ} \mathrm{C}$ a $900{ }^{\circ} \mathrm{C}$ en condiciones no isotérmicas a cuatro regímenes de calentamiento $5,10,15$ y $20{ }^{\circ} \mathrm{C} / \mathrm{min}$ respectivamente. La primera etapa de calentamiento fue de 30 ${ }^{\circ} \mathrm{C}$ a $105{ }^{\circ} \mathrm{C}$ a $5{ }^{\circ} \mathrm{C} / \mathrm{min}$ y se mantuvo en $105{ }^{\circ} \mathrm{C}$ por 15 minutos para eliminar la humedad residual de las muestras, luego se calentó de $105{ }^{\circ} \mathrm{C}$ a $900{ }^{\circ} \mathrm{C}$ según la velocidad de calentamiento deseada $\left(5,10,15\right.$ y $\left.20^{\circ} \mathrm{C} / \mathrm{min}\right)$ y cuando se 
llegó a $900{ }^{\circ} \mathrm{C}$ se mantuvo por 10 minutos. Como gas inertizante se usó nitrógeno a $60 \mathrm{~cm}^{3} / \mathrm{min}$ durante todo el proceso. Cada experimento se repitió al menos tres veces para asegurar la repetibilidad.

\section{Análisis cinético}

Considerando la conversión $\alpha$ de la muestra como:

$\alpha=\frac{m_{o}-m}{m_{o}-m_{f}}$

Donde: $\mathrm{m}_{\mathrm{o}}$ es la masa inicial de la muestra, $\mathrm{m}$ la masa en cualquier momento y $\mathrm{m}_{\mathrm{f}}$ la masa final de la muestra. Luego la descomposición térmica de la biomasa se puede expresar por:

$\frac{d \alpha}{d t}=k(T) f(\alpha)$

Siendo: T la temperatura, $f(\alpha)$ el modelo de la reacción y $k(T)$ la constante de la velocidad de la reacción expresada por la ecuación de Arrhenius:

$k(T)=A e^{-E / R T}$

Donde: A es el factor de frecuencia $\left(\mathrm{min}^{-1}\right)$, E la energía de activación $(\mathrm{kJ} / \mathrm{mol}), \mathrm{R}$ la constante universal de los gases (8.314 x $\left.10^{-3} \mathrm{~kJ} / \mathrm{K} . \mathrm{mol}\right)$ y $\mathrm{T}$ la temperatura en K. Para determinar la energía de activación empleando los datos generados por el análisis termogravimétrico hay varias aproximaciones de las cuales consideramos una aproximación isoconversional diferencial (Friedman) e isoconversional integral (Ozawa-Flynn-Wall).

1) Método de Friedman: Si se tiene que el programa de calentamiento varia linealmente según:

$\mathrm{T}=\mathrm{T}_{o}+\beta t$

Donde $\beta$ es la velocidad de calentamiento, diferenciado la ecuación (4) respecto a t y combinando con la ecuación (2) y (3) se tiene:

$\beta \frac{d \alpha}{d T}=\left(e^{-E / R T}\right)(\mathrm{A} f(\alpha))$

Tomado logaritmo natural a la ecuación (5) se tiene:

$\ln \left(\beta \frac{d \alpha}{d T}\right)_{\alpha, i}=\ln \left(A_{\alpha} f(\alpha)\right)-\frac{E}{R T_{\alpha, i}}$

Donde $\alpha$ es la conversión e i denota la velocidad de calentamiento $\beta$. Luego para un $\alpha$ cualquiera el valor de $\mathrm{E}_{\alpha}$ se puede hallar de la pendiente de la recta obtenida por graficar $\ln \left(\beta \frac{d \alpha}{d T}\right)_{\alpha, i}$ vs. $\frac{1}{T_{\alpha, i}}$ Se recomienda trabajar en el intervalo de 0.1 a 0.9 para la conversión $\alpha$ y a un paso de 0.05 . El método de Friedman tiene como ventaja que se puede aplicar no solamente a un programa de calentamiento lineal sino que podemos aplicarlo a la temperatura real de la muestra la cual se puede desviar de la temperatura establecida por el efecto térmico inducido por el proceso, su desventaja radica en que es sensible al ruido experimental de los datos termogravimétricos que son de naturaleza integral [19].
2) Método de Ozawa-Flynn-Wall $(O F W)$ : Los datos integrales son mejor tratados por métodos isoconversionales integrales, si combinamos la ecuación (2) y (3) se tiene:

$\frac{d \alpha}{f(\alpha)}=A e^{-E / R T} d t$

Integrando la ecuación (7) tenemos:

$\int_{0}^{\alpha} \frac{d \alpha}{f(\alpha)}=\int_{0}^{t} A e^{-E / R T} d t$

Como $f(\alpha)$ no se conoce, entonces $\int_{0}^{\alpha} \frac{d \alpha}{f(\alpha)}$ puede ser expresada como $g(\alpha)=\int_{0}^{\alpha} \frac{d \alpha}{f(\alpha)}$ lo que viene a ser la forma integral del modelo de reacción, acomodando la ecuación (8) queda como:

$g(\alpha)=A \int_{0}^{t} e^{-E / R T} d t$

si el programa de calentamiento es lineal y expresado por la ecuación (4), diferenciando respecto a $\mathrm{T}$ y reemplazando en la ecuación (9) se tiene:

$g(\alpha)=\frac{A}{\beta} \int_{0}^{T} e^{-E / R T} d T=\frac{A E}{\beta R} p(x)$

La integral no tiene una solución analítica y se resuelve por integración numérica o por una función de aproximación. OFW propone la aproximación de Doyle [20] para $p(x)=0.0048 e^{-1.052 E / R T}$, reemplazando en la ecuación (10) se tiene $\beta=\left(\frac{A E}{\operatorname{Rg}(\alpha)}\right)(0.00484) e^{-1.052 E / R T}$, tomando logaritmo natural y acomodando queda:

$\ln (\beta)=\ln \left(\frac{A E}{R g(\alpha)}\right)-5.331-1.052 \frac{E}{R T_{\alpha, i}}$

Entonces para un $\alpha$ cualquiera el valor de $\mathrm{E}_{\alpha}$ se puede obtener de la pendiente de la recta obtenida de graficar $\ln (\beta)$ vs. $\frac{1}{T_{\alpha, i}}$.

\section{RESULTADOS Y DISCUSIÓN}

\section{A. Análisis termogravimétrico no isotérmico}

En la Fig. 1 se identifican tres regiones, en la primera región desde la temperatura inicial hasta $120{ }^{\circ} \mathrm{C}$ se da la etapa de deshidratación y perdida parcial de volátiles, la segunda etapa se da entre $120{ }^{\circ} \mathrm{C}$ hasta $410{ }^{\circ} \mathrm{C}$ que implica la descomposición de la hemicelulosa $\left(220^{\circ} \mathrm{C}-315^{\circ} \mathrm{C}\right)$ y la celulosa $\left(315{ }^{\circ} \mathrm{C}-400{ }^{\circ} \mathrm{C}\right)$ así como el desprendimiento de compuestos volátiles, aquí ocurre la máxima descomposición de la biomasa, luego en una tercera etapa entre $410{ }^{\circ} \mathrm{C}$ a 650 ${ }^{\circ} \mathrm{C}$ se da la descomposición de la lignina, y la oxidación del biochar [21]. De la Fig. 1 (curva DTG) se obtienen las temperaturas de máxima descomposición de las biomasas estudiadas, para el BCA a $350.72{ }^{\circ} \mathrm{C}$ se ha descompuesto hasta el $55.27 \%$, para los RFV a $317.28^{\circ} \mathrm{C}$ se descompone hasta el $46.83 \%$. La biomasa que se descompone más es el BCA, seguido por los RFV. Este orden es similar al establecido por 
la relación $\mathrm{MV} / \mathrm{CF}$ expuesta en la Tabla I. Si analizamos la Fig. 2 vemos que, a los regímenes de calentamiento de 5, 10, 15 y $20{ }^{\circ} \mathrm{C} / \mathrm{min}$, todas las curvas TGA muestran un comportamiento similar con las tres regiones mencionadas anteriormente, el comportamiento es análogo para los RFV.

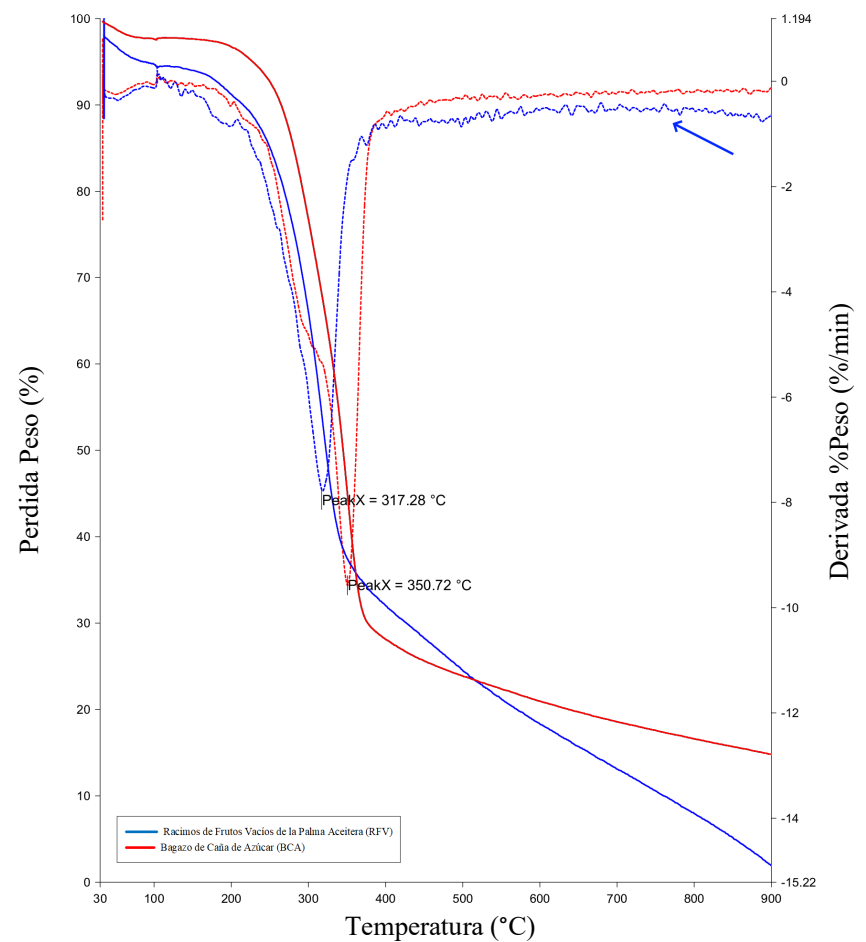

Fig. 1 Curvas TGA y DTG del Bagazo de Caña de Azúcar y de los Racimos de Frutos Vacíos de la Palama Aceitera, obtenidas a $\beta=10^{\circ} \mathrm{C} / \mathrm{min}$, flujo de $\mathrm{N}_{2}$ de $60 \mathrm{~cm}^{3} / \mathrm{min}$

\section{B. Análisis cinético}

De los resultados obtenidos por el análisis termogravimétrico se decidió trabajar en el rango de $200{ }^{\circ} \mathrm{C}$ a $700{ }^{\circ} \mathrm{C}$ para el análisis cinético empleando los métodos de Ozawa-Flynn-Wall y Friedman, para determinar la energía de activación de las muestras de biomasa y de los diferentes catalizadores evaluados.

1) Bagazo de caña de azúcar: En las Tablas IV y V se muestran los valores de la energía de activación para el rango de conversión de 0.05 a 0.80 calculado por los métodos de Ozawa-Flynn-Wall (OFW) y Friedman respectivamente. En ambos métodos el coeficiente de regresión lineal promedio $\mathrm{R}^{2}$ está entre 0.823 a 0.991 para el método de OFW y 0.807 a 0.991. En la Fig. 3 se graficó la energía de activación promedio del BCA sin y con catalizador, según los dos métodos cinéticos empleados. Si el catalizador adecuado es aquel que presenta la energía de activación promedio más baja respecto al de la biomasa sin catalizador se tiene que Z2 (catalizador de equilibrio de FCC) es el mejor según ambos métodos cinéticos. Asimismo, Z5 (zeolita HY) no es adecuada porque su energía de activación es mayor que el de la biomasa sin catalizador. Los otros catalizadores Z3, Z4 y Z6 son diferentes formas del ZSM-5 y todos presentan valores relativamente cercanos entre sí.

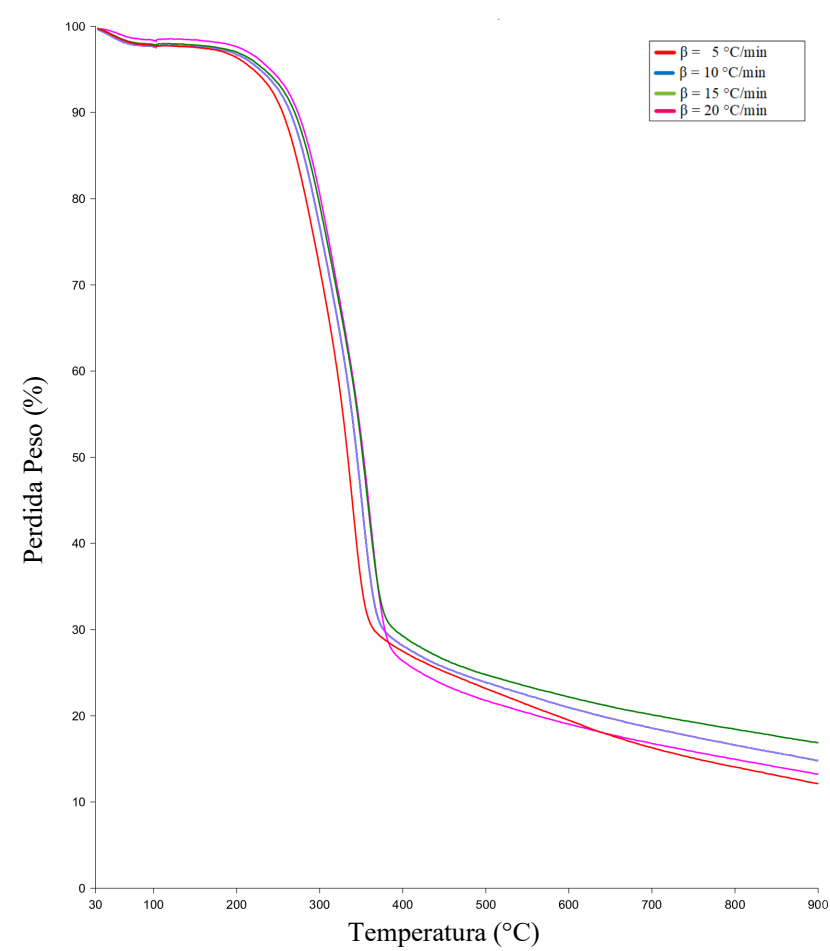

Fig. 2 Curvas TGA a diferentes velocidades de calentamiento para el Bagazo de Caña de Azúcar

TABLA IV

ENERGÍA DE ACTIVACIÓN PARA EL BCA - MÉTODO OFW

\begin{tabular}{|c|c|c|c|c|c|c|}
\hline \multirow[t]{2}{*}{$\alpha$} & \multicolumn{6}{|c|}{ Energía de Activación $(\mathrm{kJ} / \mathrm{mol})$} \\
\hline & $\mathrm{BCA}$ & $\mathrm{Z2}$ & $\mathrm{Z3}$ & $\mathrm{Z4}$ & $\mathrm{Z5}$ & Z6 \\
\hline 0.05 & 133.64 & 137.24 & 120.80 & 151.76 & 215.30 & 141.46 \\
\hline 0.10 & 124.77 & 120.96 & 113.45 & 122.04 & 147.49 & 123.00 \\
\hline 0.15 & 124.63 & 122.40 & 113.34 & 116.19 & 140.25 & 121.60 \\
\hline 0.20 & 128.16 & 124.99 & 115.68 & 116.91 & 138.70 & 122.82 \\
\hline 0.25 & 132.55 & 127.00 & 119.22 & 119.61 & 136.86 & 124.55 \\
\hline 0.30 & 136.81 & 128.89 & 121.75 & 122.09 & 135.44 & 125.43 \\
\hline 0.35 & 140.92 & 131.59 & 123.46 & 123.70 & 135.68 & 126.51 \\
\hline 0.40 & 144.64 & 131.59 & 124.92 & 125.04 & 135.79 & 128.13 \\
\hline 0.45 & 143.62 & 132.17 & 126.48 & 125.54 & 137.52 & 127.50 \\
\hline 0.50 & 145.48 & 133.56 & 127.38 & 126.15 & 140.89 & 127.73 \\
\hline 0.55 & 142.72 & 136.46 & 128.89 & 128.45 & 159.65 & 130.34 \\
\hline 0.60 & 143.15 & 140.22 & 131.99 & 133.36 & 400.01 & 134.15 \\
\hline 0.65 & 143.60 & 170.79 & 144.58 & 166.60 & 188.88 & 168.81 \\
\hline 0.70 & 143.94 & 33.55 & 165.58 & 158.23 & 194.94 & 169.83 \\
\hline 0.75 & 144.48 & 59.81 & 193.63 & 139.58 & 155.66 & 146.96 \\
\hline 0.80 & 160.05 & 140.95 & 154.38 & 121.97 & 132.48 & 129.63 \\
\hline Media & 139.57 & 123.26 & 132.84 & 131.08 & 168.47 & 134.28 \\
\hline
\end{tabular}


Para el BCA sin catalizador Ounas [7] empleando el método de OFW reporto valores de la energía de activación de $163-235 \mathrm{~kJ} / \mathrm{mol}$ en un rango de conversión de 0.2 a 0.9 y Jayaraman [22] empleando el método de Friedman reporto valores de la energía de activación entre $121-276 \mathrm{~kJ} / \mathrm{mol}$ en un rango de conversión de 0.2 a 0.6 , los valores promedios hallados en esta investigación para un rango de conversión de 0.05 a 0.80 son de $139.57 \mathrm{~kJ} / \mathrm{mol}$ aplicando el método de OFW y $147.88 \mathrm{~kJ} / \mathrm{mol}$ empleando el método de Friedman, ambos valores están en concordancia con los de las investigaciones citadas.

TABLA V

ENERGÍA DE ACTIVACIÓN PARA EL BCA - MÉTODO DE FRIEDMAN

\begin{tabular}{|c|c|c|c|c|c|c|}
\hline \multirow{2}{*}{$\alpha$} & \multicolumn{6}{|c|}{ Energía de Activación (kJ/mol) } \\
\cline { 2 - 7 } & BCA & Z2 & Z3 & Z4 & Z5 & Z6 \\
\hline 0.05 & 122.74 & 119.22 & 110.43 & 124.71 & 173.05 & 122.53 \\
\hline 0.10 & 119.91 & 113.54 & 107.86 & 106.41 & 128.94 & 113.33 \\
\hline 0.15 & 124.58 & 118.52 & 111.45 & 107.96 & 126.89 & 115.82 \\
\hline 0.20 & 131.83 & 125.02 & 116.25 & 113.56 & 130.13 & 120.17 \\
\hline 0.25 & 139.14 & 128.49 & 121.39 & 119.72 & 131.18 & 124.01 \\
\hline 0.30 & 143.45 & 129.78 & 124.50 & 123.47 & 131.29 & 125.02 \\
\hline 0.35 & 146.17 & 132.25 & 125.73 & 124.56 & 134.04 & 125.20 \\
\hline 0.40 & 146.93 & 132.94 & 126.57 & 125.64 & 140.21 & 127.21 \\
\hline 0.45 & 142.50 & 134.93 & 128.88 & 127.28 & 155.96 & 128.20 \\
\hline 0.50 & 142.40 & 142.52 & 132.90 & 131.15 & 176.36 & 131.63 \\
\hline 0.55 & 139.48 & 160.89 & 140.33 & 137.35 & 197.93 & 140.06 \\
\hline 0.60 & 139.88 & 177.07 & 147.13 & 140.40 & 460.62 & 143.37 \\
\hline 0.65 & 144.63 & 205.47 & 156.08 & 169.33 & 184.81 & 174.85 \\
\hline 0.70 & 161.74 & 3.38 & 163.75 & 152.30 & 181.04 & 167.40 \\
\hline 0.75 & 191.23 & 41.73 & 182.42 & 124.74 & 129.63 & 136.05 \\
\hline 0.80 & 229.43 & 116.78 & 131.88 & 101.36 & 112.67 & 111.48 \\
\hline Media & 147.88 & 123.91 & 132.97 & 126.87 & 168.42 & 131.65 \\
\hline
\end{tabular}

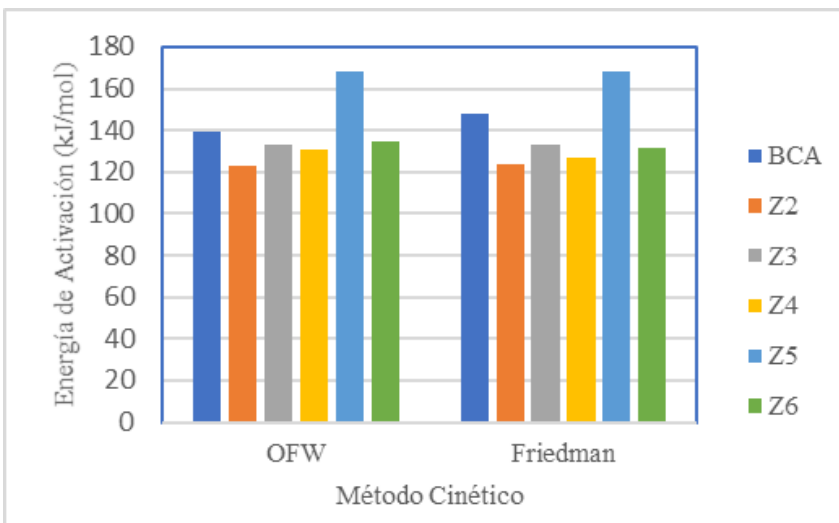

Fig. 3 Energía de activación promedio de la biomasa BCA con y sin catalizador, calculada según OFW y Friedman
2) Racimos de Frutos Vacios de Palma Aceitera (RFV): En las Tablas VI y VII se muestran los valores de la energía de activación para el rango de conversión de 0.05 a 0.80 calculado por los métodos de Ozawa-Flynn-Wall (OFW) y Friedman respectivamente. En ambos métodos el coeficiente de regresión lineal promedio $\mathrm{R}^{2}$ está entre 0.937 a 0.995 para el método de OFW y 0.880 a 0.980 para el método de Friedman, solo se están reportando valores de la energía de activación en el rango lineal y con un $\mathrm{R}^{2}$ mayor a 0.8. En la Fig. 4 se graficó la energía de activación promedio del RFV con y sin catalizador, según los dos métodos cinéticos empleados. Como la energía de activación representa la mínima energía requerida para que la reacción se inicie [14], entonces el catalizador adecuado sería el que presente la energía de activación promedio más baja respecto al de la biomasa sin catalizador, luego Z2 (catalizador de equilibrio de FCC) es el mejor según ambos métodos cinéticos, Los catalizadores Z6 (ZSM-5 polvo) y Z3 (ZSM-5 en pellet) muestran la misma tendencia en ambos análisis cinéticos, no sucede lo mismo con el Z4 (ZSM-5 en pellet esférico) el cual cumple el criterio de la energía de activación según el método de OFW, pero no según el método de Friedman, asimismo para el caso del Z5 (zeolita HY) la energía de activación es mayor que el de la biomasa sin catalizador para ambos casos.

TABLA VI

ENERGía DE ACTIVACIÓN PARA LOS RFV - MÉTODO OFW

\begin{tabular}{|c|c|c|c|c|c|c|}
\hline \multirow{2}{*}{$\alpha$} & \multicolumn{6}{|c|}{ Energía de Activación (kJ/mol) } \\
\cline { 2 - 7 } & RFV & Z2 & Z3 & Z4 & Z5 & Z6 \\
\hline 0.05 & 432.64 & N.A. & 147.95 & 189.02 & 193.18 & 136.12 \\
\hline 0.10 & 177.97 & N.A. & 136.27 & 174.94 & 165.63 & 119.45 \\
\hline 0.15 & 147.66 & 149.45 & 130.84 & 160.09 & 153.56 & 114.58 \\
\hline 0.20 & 137.68 & 132.24 & 130.04 & 153.70 & 152.80 & 115.41 \\
\hline 0.25 & 131.82 & 118.94 & 129.23 & 153.05 & 152.43 & 114.27 \\
\hline 0.30 & 125.62 & 108.04 & 129.98 & 152.37 & 154.26 & 113.45 \\
\hline 0.35 & 126.11 & 96.69 & 130.86 & 153.91 & 156.57 & 117.48 \\
\hline 0.40 & 124.12 & N.A. & 135.89 & 157.11 & 181.77 & 117.99 \\
\hline 0.45 & 124.48 & N.A. & 144.16 & 173.85 & N.A. & 122.79 \\
\hline 0.50 & 123.24 & N.A. & 168.99 & N.A. & N.A. & 129.90 \\
\hline 0.55 & 126.42 & N.A. & N.A. & N.A. & N.A. & 171.95 \\
\hline 0.60 & 127.54 & N.A. & N.A. & N.A. & N.A. & N.A. \\
\hline 0.65 & 151.22 & N.A. & N.A. & N.A. & N.A. & N.A. \\
\hline 0.70 & N.A. & N.A. & N.A. & N.A. & N.A. & N.A. \\
\hline 0.75 & N.A. & 103.60 & 109.27 & N.A. & N.A. & N.A. \\
\hline 0.80 & N.A. & 92.43 & 84.46 & 91.28 & N.A. & N.A. \\
\hline Media & 158.19 & 114.48 & 131.49 & 155.93 & 163.77 & 124.85 \\
\hline N.A. va & N E 91.85 & & \\
\hline
\end{tabular}

N.A. valores de $\mathrm{E}$ que no son lineales y con $\mathrm{R}^{2}<0.8$

Para los RFV sin catalizador Nyakuma [23] empleando el método de OFW reporto valores de la energía de activación promedio de $146.63 \mathrm{~kJ} / \mathrm{mol}$ en un rango de 
conversión de 0.1 a 0.7 , otras investigaciones aplicando los métodos de Popescu y DAEM han reportado valores promedio de la energía de activación de $137.85 \mathrm{~kJ} / \mathrm{mol}$ para un $\alpha$ de 0.05 a $0.70,147.08 \mathrm{~kJ} / \mathrm{mol}$ para un $\alpha$ de 0.05 a 0.70 y 177.33 $\mathrm{kJ} / \mathrm{mol}$ para un $\alpha$ de 0.0.1 a 0.9 ; esta investigación tiene un valor promedio de la energía de activación de $158.19 \mathrm{~kJ} / \mathrm{mol}$ para un $\alpha$ de 0.05 a 0.80 [24], [10], [5], la revisión bibliográfica realizada no ha hallado trabajos sobre la cinética de los RFV aplicando el método de Friedman pero lo reportado está en concordancia con los valores hallados según OFW para $\alpha 0.05$ a 0.65 .

TABLA VII

ENERGÍA DE ACTIVACIÓN PARA LOS RFV - MÉTODO DE FRIEDMAN

\begin{tabular}{|c|c|c|c|c|c|c|}
\hline \multirow[t]{2}{*}{$\alpha$} & \multicolumn{6}{|c|}{ Energía de Activación $(\mathrm{kJ} / \mathrm{mol})$} \\
\hline & RFV & $\mathrm{Z2}$ & Z3 & $\mathrm{Z4}$ & $\mathrm{Z5}$ & Z6 \\
\hline 0.05 & 302.02 & 59.39 & 131.68 & 175.21 & 191.78 & 119.01 \\
\hline 0.10 & 136.89 & 136.03 & 123.64 & 159.04 & 159.30 & 108.00 \\
\hline 0.15 & 119.72 & 115.62 & 121.77 & 150.57 & 152.30 & 105.48 \\
\hline 0.20 & 117.77 & 99.39 & 122.92 & 147.02 & 155.70 & 109.66 \\
\hline 0.25 & 117.60 & N.A. & 124.89 & 149.47 & 165.18 & 111.94 \\
\hline 0.30 & 114.70 & N.A. & 129.68 & 154.89 & 187.35 & 113.70 \\
\hline 0.35 & 116.93 & N.A. & 139.78 & 172.36 & 219.29 & 122.39 \\
\hline 0.40 & 118.51 & N.A. & 155.82 & 197.66 & 283.28 & 133.25 \\
\hline 0.45 & 122.00 & N.A. & 178.54 & 243.99 & N.A. & 155.49 \\
\hline 0.50 & 127.42 & N.A. & 214.94 & N.A. & N.A. & 179.79 \\
\hline 0.55 & 146.12 & N.A. & N.A. & N.A. & N.A. & 244.90 \\
\hline 0.60 & 164.09 & N.A. & N.A. & N.A. & N.A. & N.A. \\
\hline 0.65 & 201.55 & N.A. & N.A. & N.A. & N.A. & N.A. \\
\hline 0.70 & N.A. & 102.32 & N.A. & N.A. & N.A. & N.A. \\
\hline 0.75 & N.A. & N.A. & N.A. & N.A. & N.A. & N.A. \\
\hline 0.80 & N.A. & N.A. & N.A. & N.A. & N.A. & N.A. \\
\hline Media & 146.56 & 102.55 & 144.37 & 172.24 & 189.27 & 136.69 \\
\hline
\end{tabular}

N.A. valores de $\mathrm{E}$ que no son lineales y con $\mathrm{R}^{2}<0.8$

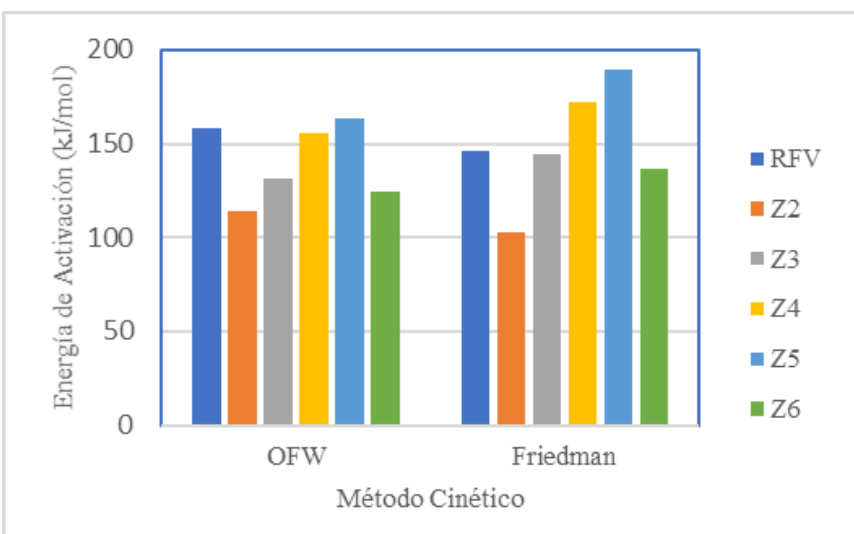

Fig. 4 Energía de activación promedio de la biomasa RFV con y sin catalizador, calculada según OFW y Friedman
Los resultados hallados de la energía de activación de las biomasas sin catalizador muestran una excelente correlación con investigaciones similares. Las energías de activación de la biomasa más el catalizador no han podido ser comparados con similares porque como afirma Yang en [25] hay muchos estudios sobre la cinética de la pirólisis de la biomasa pero hay mucho menos sobre la cinética de la pirólisis catalítica de la biomasa y en este caso particular menos sobre la pirólisis catalítica empleando zeolitas como catalizador para la pirólisis del bagazo de caña de azúcar y de los racimos de frutos vacíos de la palma aceitera, la mayoría de los trabajos se han enfocado en el efecto del catalizador sobre los rendimientos de productos líquidos, gaseosos y sólidos.

En el caso de la pirólisis catalítica del BCA el catalizador Z5 (zeolita HY) es el único con energía de activación mayor al de la biomasa sin catalizador, esto ocurre también para los RFV, pero solo para el catalizador Z4 y cuando la energía de activación es calculada por el método de Friedman. Como señala Navqi en [26] después de la descomposición inicial de la biomasa los productos líquidos alquitranados se carbonizan y ese carbono puede aumentar la energía de activación. Las zeolitas macroporosas como la $\mathrm{HY}$ (Z5) permiten una rápida difusión de los reactantes y llevan a la formación de grandes compuestos poliaromáticos que producen coque, en cambio las zeolitas mesoporosas como la ZSM-5 producen más hidrocarburos aromáticos y menos coque debido a su tamaño de poro más pequeño, lo que sugiere que un tamaño de poro pequeño impide la entrada de los grandes precursores del coque [16].

Los resultados de la energía de activación calculadas para los RFV y el catalizador Z4 aplicando el método de Friedman pueden estar influenciadas por el ruido de los datos experimentales ya que los datos son esencialmente del tipo integral [19].

\section{CONCLUSIONES}

Se investigó la cinética de la pirólisis catalítica de los racimos de frutos vacíos de la palma aceitera y del bagazo de caña de azúcar, usando el análisis termogravimétrico no isotérmico, se aplicaron dos métodos isoconversionales el método diferencial de Friedman y el método integral de Ozawa-Flynn-Wall, se evaluaron cinco catalizadores y se concluye que el catalizador de equilibrio de FCC (Z2) presenta la menor energía de activación respecto a la energía de activación de la pirólisis de la biomasa sin catalizador y por tanto sería el más útil para la pirólisis catalítica del BCA y de los RFV. La zeolita HY (Z5) es el catalizador con la mayor energía de activación y el menos útil para el proceso de pirólisis catalítica.

El análisis cinético por métodos isoconversionales integrales aplicado a los datos termogravimétricos no isotérmicos permite determinar de modo fiable las energías de activación en la pirólisis con y sin catalizador de las biomasas lignocelulósicas. 
En general todos los catalizadores del tipo ZSM-5 mostraron energías de activación similares lo que indica que potencialmente todos ellos podrían ser útiles en el proceso de pirólisis catalítica.

\section{AGRADECIMIENTOS}

Los autores agradecen a la Unidad de Investigación de la Facultad de Ingeniería de Petróleo, Gas Natural y Petroquímica de la UNI por el apoyo prestado para la realización de este proyecto.

\section{REFERENCIAS}

[1] British Petroleum, "BP Energy Outlook 2019 edition The Energy Outlook explores the forces shaping the global energy transition out to 2040 and the key uncertainties surrounding that," 2019.

2] Ministerio de Agricultura y Riego, "Serie de Estadísticas de Producción Agrícola," Serie de Estadisticas de Producción Agrícola (SEPA), 2020. [Online]. Available:

http://frenteweb.minagri.gob.pe/sisca/?mod=consulta cult. [Accessed: 15-Jan-2020].

[3] V. S. Y. Soon, B. L. F. Chin, and A. C. R. Lim, "Kinetic study on pyrolysis of oil palm frond," IOP Conf. Ser. Mater. Sci. Eng., vol. 121, no. 1, 2016, doi: 10.1088/1757-899X/121/1/012004.

4] K. G. Santos, T. S. Lira, V. V. Murata, M. Gianesella, and M. A. S. Barrozo, "Pyrolysis of sugarcane bagasse: A consecutive reactions kinetic model from TGA experiments," Mater. Sci. Forum, vol. 660-661, pp. 593-598, 2010, doi:

10.4028/www.scientific.net/MSF.660-661.593.

[5] F. Surahmanto, H. Saptoadi, H. Sulistyo, and T. A. Rohmat, "Investigation of the slow pyrolysis kinetics of oil palm solid waste by the distributed activation energy model," Biofuels, vol. 0, no. 0 , pp. 1-8, 2017, doi: 10.1080/17597269.2017.1387750.

6] Z. Chen et al., "Characteristics and kinetic study on pyrolysis of five lignocellulosic biomass via thermogravimetric analysis," Bioresour. Technol., vol. 192, pp. 441-450, 2015, doi:

10.1016/j.biortech.2015.05.062.

[7] A. Ounas, A. Aboulkas, K. El harfi, A. Bacaoui, and A. Yaacoubi, "Pyrolysis of olive residue and sugar cane bagasse: Non-isothermal thermogravimetric kinetic analysis," Bioresour. Technol., vol. 102, no. 24, pp. 11234-11238, 2011, doi:

10.1016/j.biortech.2011.09.010.

[8] X. Chen et al., "Recent developments in lignocellulosic biomass catalytic fast pyrolysis: Strategies for the optimization of bio-oil quality and yield," Fuel Process. Technol., vol. 196, no. August, 2019, doi: 10.1016/j.fuproc.2019.106180

[9] A. Strubinger, F. Morales, and K. Aponte, "Environmental risk and use of FCC spent catalyst in mortars mixtures," Rev. la Fac. Ing., vol. 29, no. 4, pp. 93-106, 2014.

[10] B. B. Nyakuma, A. Ahmad, A. Johari, and T. A. T. Abdullah, "Thermogravimetric and kinetic analyses of oil palm empty fruit bunch (OPEFB) Pellets using the distributed activation energy model," J. Phys. Sci. vol. 27, no. 3, pp. 67-83, 2016, doi: 10.21315/jps2016.27.3.5

[11] P. Luangkiattikhun, C. Tangsathitkulchai, and M. Tangsathitkulchai, "Non-isothermal thermogravimetric analysis of oil-palm solid wastes," Bioresour. Technol., vol. 99, no. 5, pp. 986-997, 2008, doi: 10.1016/j.biortech.2007.03.001.

[12] S. Vyazovkin et al., "ICTAC Kinetics Committee recommendations for collecting experimental thermal analysis data for kinetic computations," Thermochim. Acta, vol. 590, pp. 1-23, 2014, doi 10.1016/j.tca.2014.05.036

[13] S. Vyazovkin, A. K. Burnham, J. M. Criado, L. A. Pérez-Maqueda, C. Popescu, and N. Sbirrazzuoli, "ICTAC Kinetics Committee recommendations for performing kinetic computations on thermal analysis data," Thermochim. Acta, vol. 520, no. 1-2, pp. 1-19, 2011, doi: 10.1016/j.tca.2011.03.034.

[14] Z. Ma, D. Chen, J. Gu, B. Bao, and Q. Zhang, "Determination of pyrolysis characteristics and kinetics of palm kernel shell using TGA-FTIR and model-free integral methods," Energy Convers. Manag., vol. 89, pp. 251-259, 2015, doi: 10.1016/j.enconman.2014.09.074.

[15] A. K. Varma and P. Mondal, "Physicochemical Characterization and Pyrolysis Kinetic Study of Sugarcane Bagasse Using Thermogravimetric Analysis," J. Energy Resour. Technol., vol. 138, no. 5, pp. 1-11, 2016, doi: 10.1115/1.4032729.

[16] M. M. Rahman, R. Liu, and J. Cai, "Catalytic fast pyrolysis of biomass over zeolites for high quality bio-oil - A review," Fuel Process. Technol., vol. 180, no. June, pp. 32-46, 2018, doi: 10.1016/j.fuproc.2018.08.002.

[17] V. Pretell and R. Erazo, "Evaluation of the Physicochemical and Thermogravimetric Properties of Three Peruvian Biomasses for their Energetic use," in Industry, Innovation, and Infrastructure for Sustainable Cities and Communities: Proceedings of the 17th LACCEI International Multi-Conference for Engineering, Education and Technology, 2019, no. July 2019, pp. 24-26, doi: http://dx.doi.org/10.18687/LACCEI2019.1.1.282.

[18] D. Uriarte, "Experimentación de la obtención de combustibles líquidos a partir del polietileno de alta densidad," Universidad Nacional de Ingenieria, 2018.

[19] S. Vyazovkin, Isoconversional Kinetics of Thermally Stimulated Processes. Springer International Publishing, 2015.

[20] C. D. Doyle, "Kinetic Analysis of Thermogravimetric Data," J. Appl. Polym. Sci., vol. 5, no. 15, pp. 285-292, 1961, doi doi.org/10.1002/app.1961.070051506.

[21] M. A. A. Mohammed, A. Salmiaton, W. A. K. G. Wan Azlina, and M. S. Mohamad Amran, "Gasification of oil palm empty fruit bunches: A characterization and kinetic study," Bioresour. Technol., vol. 110, pp. 628-636, 2012, doi: 10.1016/j.biortech.2012.01.056.

[22] K. Jayaraman, I. Gokalp, S. Petrus, V. Belandria, and S. Bostyn, "Energy recovery analysis from sugar cane bagasse pyrolysis and gasification using thermogravimetry, mass spectrometry and kinetic models," J. Anal. Appl. Pyrolysis, vol. 132, no. February 2017, pp. 225-236, 2018, doi: 10.1016/j.jaap.2018.02.003.

[23] B. B. Nyakuma, S. Wong, and O. Oladokun, "Non-oxidative thermal decomposition of oil palm empty fruit bunch pellets: fuel characterisation, thermogravimetric, kinetic, and thermodynamic analyses," Biomass Convers. Biorefinery, 2019, doi 10.1007/s13399-019-00568-1.

[24] B. B. Nyakuma et al., "Kinetic analysis of Oil Palm Empty Fruit Bunch (OPEFB) pellets as Feedstock for Pyrolysis," PeerJ Prepr. vol. 3, no. e1150v1, pp. 1-8, 2015, doi: dx.doi.org/10.7287/peerj.preprints.1150v1.

[25] H. Yang, G. Ji, P. T. Clough, X. Xu, and M. Zhao, "Kinetics of catalytic biomass pyrolysis using Ni-based functional materials," Fuel Process. Technol., vol. 195, no. March, p. 106145, 2019, doi: 10.1016/j.fuproc.2019.106145.

[26] S. R. Naqvi, Y. Uemura, N. Osman, and S. Yusup, "Kinetic study of the catalytic pyrolysis of paddy husk by use of thermogravimetric data and the Coats-Redfern model," Res. Chem. Intermed., vol. 41, no. 12, pp. 9743-9755, 2015, doi: 10.1007/s11164-015-1962-0. 